\title{
Flora da Usina São José, Igarassu, Pernambuco: Aristolochiaceae e Piperaceae
}

\author{
Flora of Usina São José, Igarassu, Pernambuco: Aristolochiaceae and Piperaceae
}

Aline Melo ${ }^{1,2}$, Ariclenes A.M. Araújo ${ }^{1}$ \& Marccus Alves $^{1}$

\begin{abstract}
Resumo
Piperales compreende cinco famílias, cerca de 25 gêneros e 4000 espécies, das quais apenas Aristolochiaceae e Piperaceae estão presentes no Nordeste do Brasil. Este trabalho é parte da série de monografias taxonômicas de famílias encontradas nos fragmentos de Floresta Atlântica da Usina São José, Igarassu, Pernambuco, Brasil. Coletas foram realizadas em nove fragmentos, entre os anos de 2007 e 2011, além de visita a herbários. Foram reconhecidos nove táxons: Aristolochia labiata Willd., A. papillaris Mast. (Aristolochiaceae); Peperomia magnoliifolia (Jacq.) A. Dietr., P. pellucida (L.) Kunth., Piper arboreum var. hirtellum Yunck., P. caldense C. DC., P. hispidum Sw., P. hostmannianum (Miq.) C.DC. e P. marginatum Jacq. (Piperaceae). São apresentados chave de identificação, descrições, comentários e ilustrações. Piper hostmannianum tem aqui seu primeiro registro para o Nordeste.

Palavras-chave: Floresta Atlântica, Aristolochia, Região Neotropical, novos registros, Piperales.
\end{abstract}

\begin{abstract}
Piperales comprises five families, approximately 25 genera and 4000 species, with only Aristolochiaceae and Piperaceae occurring in Northeastern Brazil. This study is include in a serie of taxonomic monographs of the families found in the Atlantic forest fragments of the Usina São José, Igarassu, Pernambuco, Brazil. Field trips were conducted in nine fragments, between 2007 and 2011, and vouchers from herbaria were also studied. Nine taxa were found: Aristolochia labiata Willd., A. papillaris Mast. (Aristolochiaceae); Peperomia magnoliifolia (Jacq.) A. Dietr., P. pellucida (L.) Kunth., Piper arboreum var. hirtellum Yunck., P. caldense C. DC., P. hispidum Sw., P. hostmannianum (Miq.) C.DC. and $P$. marginatum Jacq. (Piperaceae). Identification keys, descriptions, comments and illustrations of the species are presented. Piper hostmannianum is recorded for the first time in Northeastern of Brazil.
\end{abstract}

Key words: Atlantic Forest, Aristolochia, Neotropics, new records, Piperales.

\section{Introdução}

Piperales está inserida no clado das Magnoliids e compreende cinco famílias, Aristolochiaceae, Hydnoraceae, Lactoridaceae, Piperaceae e Saururaceae (APG III 2009; Reveal 2012), com cerca de 25 gêneros e 4000 espécies. Grande parte das espécies está em Aristolochiaceace e Piperaceae, ambas as famílias presentes no Nordeste do Brasil (Huber 1993; Tebbs 1993; Barros \& Araújo 2012; Guimarães et al. 2012).

Aristolochiaceae, com quatro gêneros e aproximadamente 500 espécies (González 1999), tem distribuição cosmopolita, embora seja predominantemente tropical e subtropical (Caperalli Jr. 2002; González 2004). No Brasil ocorre apenas Aristolochia L. com 87 espécies (Barros \& Araújo 2012). São em sua maioria plantas escandentes, geralmente lenhosas, raramente arbustivas, com folhas simples e alternas. As flores geralmente são solitárias, mas podem estar organizadas em inflorescências cimosas ou racemosas e em geral zigomorfas com o perianto subdividido em utrículo, tubo e limbo. Os verticilos reprodutivos na maioria das espécies é um conjunto tubular do androceu e gineceu denominado ginostêmio. O fruto é seco do tipo cápsula, septicida, com deiscência característica adquirindo uma forma de cesta e expondo as sementes (Hoehne 1942; Huber 1993). Também são caracterizadas por serem sapromiofílicas e pelas relações ecológicas com membros da ordem Lepidoptera (Barroso et al. 1978; Huber 1993; González 2004). No âmbito da medicina popular, são conhecidas pelo uso no tratamento de inflamações, hipotensão sanguínea, convulsões musculares e na indução de aborto (González 2004).

\footnotetext{
${ }^{1}$ Universidade Federal de Pernambuco, Depto. Botânica, Av. Prof. Moraes Rego 1235, Cidade Universitária, 50670-901, Recife, PE, Brasil.

${ }^{2}$ Autor para correspondência: aline_vmelo@yahoo.com.br
} 
Piperaceae possui cerca de 3.600 espécies em cinco gêneros, com ocorrência pantropical (Tebbs 1993; Quijano-Abril et al. 2006; Wanke 2006; Samain et al. 2008). No Brasil ocorrem três gêneros e aproximadamente 450 táxons (Guimarães et al. 2012), sendo bem representado no sub bosque de florestas (Nee 2004). São ervas terrestres, epífitas, rupícolas ou saxícolas, arbustos, arvoretas ou trepadeiras, com folhas alternas, opostas ou verticiladas, pecioladas ou não. As inflorescências estão organizadas em espiga ou racemo, solitárias ou não, terminais, axilares ou opostas às folhas. As flores são diminutas, aclamídeas, bracteadas e em geral bissexuadas. Os frutos são drupas ou bagas (Tebbs 1993; Nee 2004). Apresenta grande importância farmacêutica e química com espécies utilizadas na medicina popular como analgésicos ou antiinflamatórios (Di Stasi et al. 2002) e inseticidas naturais (Silva et al. 2007; Lima et al. 2009; Castro et al. 2010).

O objetivo deste trabalho foi inventariar e caracterizar morfologicamente as espécies de Aristolochiaceae e Piperaceae da Usina São José, Igarassu, Pernambuco, contribuindo para o conhecimento da flora local. Este trabalho é parte da série de monografias taxonômicas das famílias ocorrentes na área (Alves-Araújo \& Alves 2010; Melo et al. 2010; Pontes \& Alves 2010; Amorim \& Alves 2011; Buril \& Alves 2011; Gomes-Costa \& Alves 2012; Oliveira \& Alves 2012; Pessoa \& Alves 2012; Silva et al. 2012; Araújo \& Alves 2013).

\section{Materiais e Métodos}

A Usina São José (USJ) localiza-se no litoral norte de Pernambuco, a cerca de $40 \mathrm{~km}$ de Recife ( $7^{\circ} 40$ '21,25"- $7^{\circ} 55^{\prime}$ '50,92'S, 34 54'14,25"'$\left.35^{\circ} 05^{\prime} 21,08^{\prime \prime} \mathrm{W}\right)$, com área total de $280 \mathrm{~km}^{2}$ nos municípios de Igarassu, Abreu e Lima, Araçoiaba, Goiana, Itapissuma e Itaquitinga (Trindade et al. 2008). Está situada dentro do domínio da Mata Atlântica com vegetação de Floresta Estacional Semidecidual de Terras Baixas, com cerca de 100 fragmentos florestais entre 30 e 400 ha (Trindade et al. 2008; Alves-Araújo \& Alves 2010).

As coletas foram realizadas entre 2007 e 2011, concentradas em nove fragmentos previamente selecionados, no qual também foram alvo de estudos florísticos realizados para o local (Alves-Araújo et al. 2008; Alves-Araújo \& Alves 2010; Araújo \& Alves 2010; Melo et al. 2010;
Pontes \& Alves 2010; Amorim \& Alves 2011; Buril \& Alves 2011; Melo et al. 2011; Gomes-Costa \& Alves 2012; Oliveira \& Alves 2012;Pessoa \& Alves 2012; Silva et al. 2012; Araújo \& Alves 2013). As amostras foram submetidas às técnicas usuais em taxonomia (Mori et al. 1985) e vouchers depositados no herbário UFP, com duplicatas enviadas para o IPA, JPB e NY.

Foi realizado o levantamento das amostras de ambas as famílias depositadas nos herbários ASE, EAC, EAN, HST (Herbário Sérgio Tavares, Universidade Federal Rural de Pernambuco, não indexado), IAN, INPA, IPA, JPB, MAC, MG, PEUFR, R, RB, UFP e UFRN. As identificações e os comentários sobre distribuição geográfica foram realizados através de bibliografia especializada (Hoehne 1942; Trelease \& Yuncker 1950; Yuncker 1972, 1973, 1974; Steyermark \& Callejas-Posada 2003; Barros \& Araújo 2012; Guimarães et al. 2012) e comparação com material depositado nos acervos botânicos. São apresentadas chave de identificação, descrições e ilustrações dos caracteres diagnósticos das espécies encontradas na área de estudo.

\section{Resultados e Discussão}

Na Usina São José foram reconhecidos nove táxons pertencentes à Piperales, sendo dois de Aristolochiaceae: Aristolochia labiata Willd., A. papillaris Mast.; e sete de Piperaceae: Peperomia magnoliifolia (Jacq.) A. Dietr., P. pellucida (L.) Kunth., Piper arboreum var. hirtellum Yunck., $P$. caldense C. DC., P. hispidum Sw., P. hostmannianum (Miq.) C.DC. e P. marginatum Jacq. Os táxons de Aristolochiaceae foram observados principalmente na borda dos fragmentos e de Piperaceae encontrados tanto na borda quanto no interior dos remanescentes. Estão geralmente amplamente distribuídos na América do Sul ou Região Neotropical, com apenas duas delas sendo endêmicas para o Brasil (Aristolochia papilaris e Piper caldense). No Brasil, a maioria dos táxons encontrados na área de estudo está presente nos domínios da Mata Atlântica e Cerrado, mas também podendo ocorrer na Caatinga ou Floresta Amazônica. Neste trabalho, quatro espécies são novos registros para estados do Nordeste: Peperomia pellucida está sendo registrada para o Maranhão, Paraíba e Sergipe, Piper caldense para o Rio Grande do Norte, P. hispidum para a Paraíba e $P$. hostmannianum pela primeira vez é registrada no Nordeste. 
Chave de identificação para Piperales da Usina São José, Igarassu, PE

1. Lianas com flores solitárias e fortemente zigomorfas

Aristolochiaceae

2. Pseudoestípulas presentes; flores bilabiadas; papilas ausentes no lábio superior; fruto $10-11 \times$ 4-4,5 cm; sementes romboides, ca. 1,5 × 1,1 cm 1. Aristolochia labiata

2'. Pseudoestípulas ausentes; flores unilabiadas; papilas presentes no lábio superior; fruto ca. $4 \times 1,2$ $\mathrm{cm}$; sementes deltoides, ca. $0,5 \times 0,4 \mathrm{~cm}$ 2. Aristolochia papillaris

1'. Ervas (terrestres ou epífitas) ou arbustos (eretos ou escandentes) com flores diminutas e actinomorfas, organizadas em espiga Piperaceae

3. Ervas terrestres ou epífitas, pecíolo cilíndrico Peperomia

4. Epífitas, folhas 8-13,5 × 3,7-6 cm, obovadas a elípticas, coriáceas quando secas 3. Peperomia magnoliifolia

4'. Terrestres, folhas 1,7-3 × 1.5-2,6 cm, cordadas a deltoides, membranáceas quando secas .... 4. Peperomia pellucida

3'. Arbustos, pecíolo alado, ou quando cilíndrico sulcado na base

5. Folhas de base simétrica, cordadas, venação palmada Piper

5'. Folhas de base assimétrica, elípticas ou ovadas, venação pinada 9. Piper marginatum

6. Pecíolo alado, base da folha com diferença $1-1,8 \mathrm{~cm}$ entre os lobos

5. Piper arboreum var. hirtellum

6'. Pecíolo sulcado na base, base da folha com diferença de até $0,6 \mathrm{~cm}$ entre os lobos .. 7

7. Folhas glabras; inflorescência $2,5-3 \mathrm{~cm}$ compr. 6. Piper caldense

7'. Folhas pilosas, hirtas ou hirtelas ao menos ao longo das nervuras; inflorescência $5,3-12 \mathrm{~cm}$ compr.

8. Face adaxial da folha áspera e opaca quando seca, face abaxial hirtela 7. Piper hispidum

8'. Face adaxial da folha lisa e lustrosa quando seca, face abaxial pilosa 8. Piper hostmannianum

\section{Aristolochia L.}

Em geral lianas. Folhas predominantemente membranáceas, cordadas ou sagitadas, nervação palmada-reticulada, 5-7 nervuras primárias, margem inteira. Flores solitárias, zigomorfas, monoclamídeas, gamotépalas, perianto em utrículo, tubo e limbo, ginostêmio presente, ovário ínfero. Frutos secos, cápsulas septicidas. Sementes laminares.

Aristolochia tem distribuição cosmopolita, mas é predominantemente tropical e subtropical (Caperalli Jr. 2002; González 2004). No Brasil ocorrem 87 espécies, sendo 29 no Nordeste e cinco em Pernambuco (Barros \& Araújo 2012).

1. Aristolochia labiata Willd., Mém. Soc. Imp. Naturalistes Moscou 2: 101-102. 1809. Aristolochia brasiliensis Mart., Nov. Gen. Sp. Pl. 1: 77. 1824. syn. Aristolochia galeata Mart., Nov. Gen. Sp. Pl. 1: 76 1824. syn.

Fig. 1a,b

Ramos glabros; pseudoestípulas orbiculares, auriculares. Folhas com ápice de agudo a obtuso, glabras; pecíolo 6,5-13 com compr., glabro.
Flores com a face externa do perianto glabra; utrículo ca. $6,5 \times 4,5 \mathrm{~cm}$; tubo ca. 1,7 cm compr.; limbo bilabiado, papilas e barbelas ausentes, lábio superior oblongo, ca. $8,5 \times 12,5 \mathrm{~cm}$, base uguiculada, ápice retuso, face interna glabra, lábio inferior lanceolado, ca. 13,5 $\times 3 \mathrm{~cm}$, ápice agudo, face interna serícea, pedicelo+ovário ca. $10 \mathrm{~cm}$ compr. Cápsulas 10-11 × 4-4,5 cm, rostro ca. 0,3 cm compr.; sementes romboides, ca. 1,5 × 1,1 cm. Material examinado: Engenho D'água, 15.V.2003, fl. e fr., A. Melquíades 199 (PEUFR); XI.1995, fl., M. Alves (UFP 11572); Mata de Piedade, 19.VI.2009, fr., J.A.N. Souza 512 (UFP); 17.VI.2011, fr., A.A.M. Araújo 315 (UFP).

Amplamente distribuída na América do Sul (Pfeifer 1966). No Brasil, ocorre ao longo de quase todo o litoral, do Ceará ao Rio Grande do Sul, e em formações de Cerrado e Mata Atlântica (Barros \& Araújo 2012). Na Usina São José foi encontrada em dossel e sub-bosque, podendo ser vegetativamente reconhecida pelas pseudoestípulas orbiculares e auriculadas e pelo tom opaco da face abaxial da lâmina foliar. 

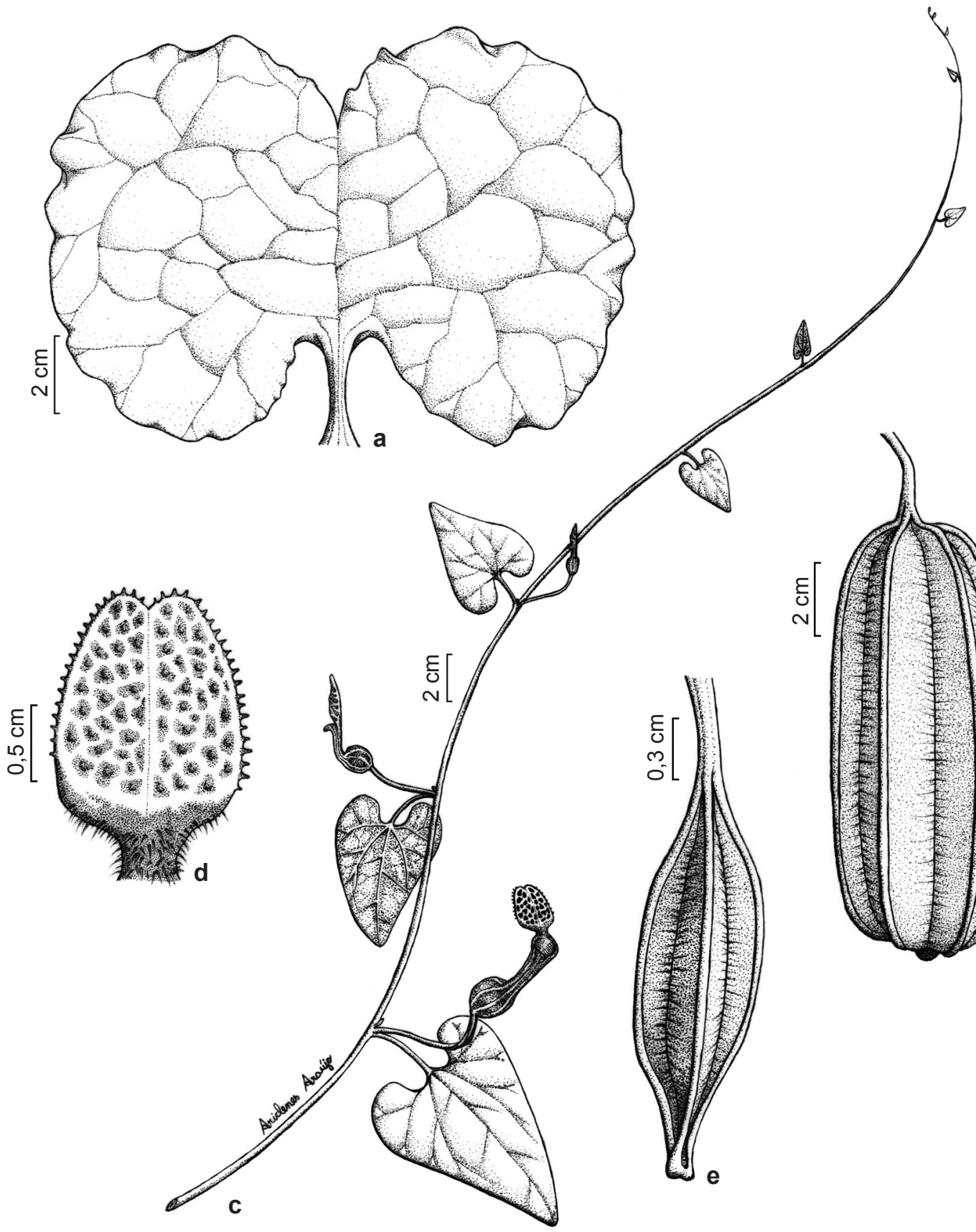

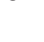

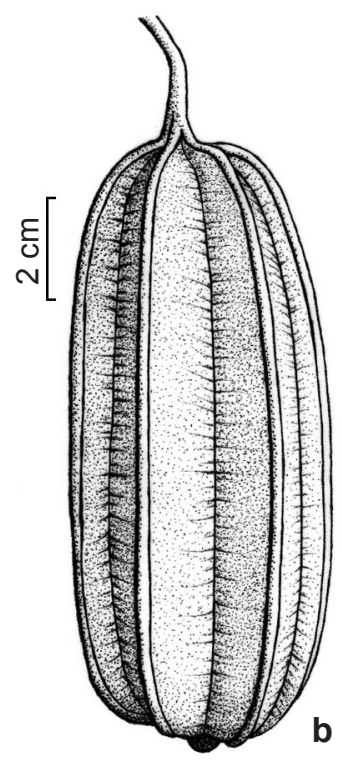

Figura 1 - a-b. Aristolochia labiata Willd - a. lábio superior do perianto; b. fruto. c-e. Aristolochia papillaris Mast - c. ramo florido; d. lábio superior do perianto; e. fruto. (a. Melquíades 199; b. Araújo 315; c-e. Araújo 354). Figura 1 - a-b. Aristolochia labiata Willd - a. upper lip of the perianth; b. fruit. c-e. Aristolochia papillaris Mast - c. flowering branch; d. upper lip of the perianth; e. fruit. (a. Melquíades 199; b. Araújo 315; c-e. Araújo 354).

2. Aristolochia papillaris Mast. in Mart., Fl. bras. 4(2): 100. 1875.

Fig. 1c-e

Ramos pubescentes quando jovens; pseudoestípulas ausentes. Folhas com ápice de agudo a cuneado, face adaxial glabra, face abaxial pubescente; pecíolo 2-2,8 cm compr., pubescente. Flores com a face externa do perianto glabrescente; utrículo ca. 1,6 $\times 1,3 \mathrm{~cm}$; tubo ca. $2 \mathrm{~cm}$ compr.; limbo unilabiado, papilas presentes, negrescentes, barbelas ausentes, lábio superior oblongo-ovalado, 
ca. 1,7 $\times 1,1 \mathrm{~cm}$, base ligeiramente mais estreita que o restante do lábio, ápice retuso, face interna glabra, setulosa apenas na base, pedicelo+ovário 2,8-3,7 $\mathrm{cm}$ compr. Cápsulas ca. $4 \times 1,2 \mathrm{~cm}$, rostro ca. 0,8 $\mathrm{cm}$ compr; sementes deltoides, ca. 0,5 $\times 0,4 \mathrm{~cm}$. Material examinado: 21.XI.2002, fl. e fr., G.J. Bezerra 108 (PEUFR); Mata de Chave, 18.XII.2009, fl., E. Pessoa 227 (UFP); Mata dos Macacos, 23.XII.2011, fl. e fr., A.A.M. Araújo 354 (UFP); Mata do Pezinho, 11.I.2008, fl., D. Araújo et al. 602 (IPA).

Espécie endêmica do Brasil, ocorrendo no Nordeste em Mata Atlântica e Cerrado, além do estado do Pará (Barros \& Araújo 2012). Na Usina São José é comumente encontrada ao longo das bordas dos fragmentos de mata, nos diversos extratos da vegetação, podendo ser reconhecida pelas flores unilabiadas e ausência de pseudoestípula.

Peperomia Ruiz \& Pav., Fl. peruv. Prodr.: 8. 1794.

Ervas terrestres ou epífitas; ramos eretos ou prostrados, glabros. Folhas alternas, cordadas a deltoides, elípticas a obovadas, base simétrica, membranáceas ou coriáceas quando secas, glândulas ausentes, glabras; pecíolo cilíndrico, glabro a piloso. Inflorescências em espigas axilares ou terminais, solitárias, eretas; raque glabra. Flores congestas ou laxas, bráctea floral orbicular a elíptica, peltada, glabra, papilosa ou não, margem eciliada; estames-2, estigma-1, apical ou subapical. Frutos drupas, globosas ou elípticas, ápice arredondado ou agudo e curvado.

Pantropical e com cerca de 1600 espécies (Quijano-Abril et al. 2006). Segundo Guimarães et al. (2012), 160 espécies estão presentes no Brasil, sendo 41 no Nordeste e nove em Pernambuco.

\section{Peperomia magnoliifolia (Jacq.) A. Dietr., Sp.} pl. 1: 153. 1831.

Fig. 2a-c

Ervas epífitas, $10-15 \mathrm{~cm}$ alt.; ramos prostrados. Folhas 8-13,5 × 3,7-6 cm, obovadas a elípticas, base acuminada, ápice arredondado ou raro emarginado, coriáceas quando secas, venação pinada, com 6 a 8 nervuras secundárias imperceptíveis e desenvolvidas ao longo da nervura mediana; pecíolo 1,5-3,5 cm compr., glabro a esparsamente piloso. Espigas 7-16 cm compr., axilares; pedúnculo 1-2 cm compr., glabro. Flores congestas, bráctea floral orbicular, papilosa exceto na margem, estigma subapical. Drupas com até 1 $\times 0,5 \mathrm{~mm}$, elípticas, papilosas; ápice ca. $0,2 \mathrm{~mm}$ compr., agudo e curvado.
Material examinado: Mata de Piedade, 29.VII.2007, fl., A. Alves-Araújo et al. 484 (UFP); 29.VII.2007, fl., $A$. Melo et al. 104 (UFP); 10.I.2008, fr., A. Alves-Araújo et al. 784 (UFP).

Presente na América Central, Caribe, Colômbia, Venezuela, Guiana, Suriname, Guiana Francesa e Brasil (Yuncker 1974; Steyermark \& Callejas-Posada 2003). No Brasil ocorre na Amazônia e Mata Atlântica, e no Nordeste nos estados do Maranhão, e de Pernambuco à Bahia (Mendes et al. 2010; Guimarães et al. 2012). Na USJ foi encontrada apenas no interior de um fragmento e distingue-se das demais pelo hábito epifítico, as folhas coriáceas quando secas, obovadas a elípticas e com ápice arredondado ou raro emarginado.

4. Peperomia pellucida (L.) Kunth., Nov. gen. sp. 1: 64. 1816.

Fig. 2d-f

Ervas terrestres de até $30 \mathrm{~cm}$ alt., ramificadas; ramos eretos. Folhas 1,7-3 × 1,5-2,6 cm, cordadas a deltoides, base cordada ou às vezes truncada, ápice agudo, membranáceas quando secas, venação palmada, 6-8 nervuras, com até 4 nervuras de menor calibre partindo ao longo da nervura mediana; pecíolo 0,5-1,5 cm compr., glabro. Espigas $2-4 \mathrm{~cm}$ compr., axilares ou terminais; pedúnculo $2-5 \mathrm{~mm}$ compr., glabro. Flores laxas, bráctea floral orbicular a elíptica, não papilosa, estigma apical. Drupas de até $0,5 \times 0,5 \mathrm{~mm}$, globosas, com estrias longitudinais.

Material examinado: Mata de Piedade, 29.VII.2007, fl. e fr., A. Alves-Araújo et al. 485 (UFP); 5.IX.2007, fr., A. Melo et al. 133 (UFP); 19.XII.2007, fr., A. Alves-Araújo et al. 721 (UFP); Mata dos Macacos, 30.IV.2008, fl., N.A. Albuquerque et al. 639 (IPA).

Material adicional examinado: BRASIL. MARANHÃO: Ribeirão, Anajatuba, 24.III.2007, fr., $M$. Ribeiro 11 (IAN). PARAÍBA: João Pessoa, 28.IX.1971, fl., I. Correia 32 (JPB). SERGIPE: Aracajú, Parque Municipal Augusto Franco, 30.VII. 2006, fl. e fr, A.K. Lima (IPA 70289).

Pantropical, sendo no Brasil presente na Amazônia, Caatinga e Floresta Atlântica, e no Nordeste nos estados do Ceará, Pernambuco, Alagoas e Bahia (Trelease \& Yuncker 1950; Lemos et al. 2010; Guimarães et al. 2012). Neste trabalho, a espécie tem distribuição ampliada para os estados do Maranhão, Paraíba e Sergipe. Conhecida popularmente como língua-de-sapo ou coraçãode-vidro, é utilizada como diurética, hipotensora, antibacteriana e analgésica (Di Stasi et al. 2002). Na área de estudo há registros em área de borda, sendo de fácil identificação por serem ervas terrestres, delicadas e pelas folhas cordadas a deltoides. 

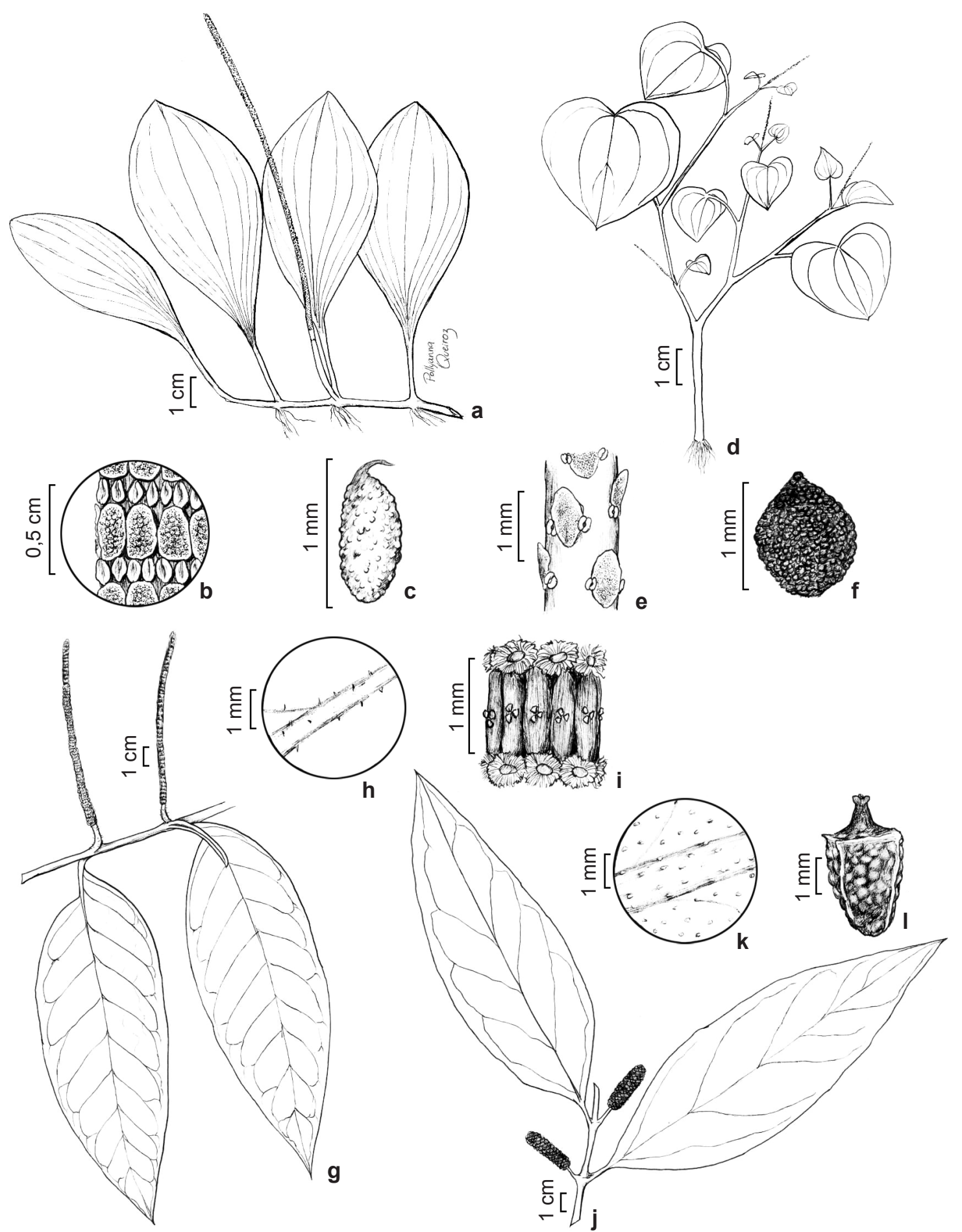

Figura 2 - a-c. Peperomia magnoliifolia (Jacq.) A. Dietr - a. hábito; b. flores; c. fruto. d-f. Peperomia pellucida (L.) Kunth - d. hábito; e. flores; f. fruto. g-i. Piper arboreum var. hirtellum Aubl. - g. ramo; h. face abaxial da folha; i. frutos. j-1. Piper caldense C. DC. -j. ramo; k. face abaxial da folha; 1 fruto. (a-c. Alves-Araújo 784; d-f. Alves-Araújo 721; g-i. Alves-Araújo 866; j-1. Gomes 312). Figura 2 - a-c. Peperomia magnoliifolia (Jacq.) A. Dietr. - a. habit; b. flowers; c. fruit. d-f. Peperomia pellucida (L.) Kunth - d. habit; e. flowers; f. fruit. g-i. Piper arboreum hirtellum Aubl. - g. shoot; h. beneath surface; i. fruits. j-1. Piper caldense C. DC. - j. shoot; k. beneath surface; 1. fruit. (a-c. Alves-Araújo 784; d-f. Alves-Araújo 721; g-i. Alves-Araújo 866; j-1. Gomes 312). 
Piper L., Sp. pl. 1: 28-30. 1753.

Arbustos eretos ou escandentes; ramos glabros, hirtos ou pilosos. Folhas alternas, elípticas, ovadas a cordadas, base assimétrica ou simétrica, membranáceas a cartáceas quando secas, glândulas presentes ou ausentes, glabras, pilosas, hirsutas ou hirtas; pecíolo alado ou cilíndrico e ao menos sulcado na base. Inflorescências em espigas, opositifolias, solitárias, eretas ou arqueadas; raque glabra ou pilosa. Flores congestas, bráctea floral orbicular a triangular, peltada, margem fimbriada; estames-4, estigmas-3, sésseis ou não, apicais, persistentes no fruto. Frutos drupas, oblongas ou globosas.

Gênero pantropical com cerca de 2000 espécies (Wanke et al. 2006) e amplamente distribuído no Brasil com 285 espécies. No Nordeste está presente com cerca de 100 táxons, sendo 17 deles registrados para Pernambuco (Guimarães et al. 2012).

5. Piper arboreum var. hirtellum Yunck., Ann. Missouri Bot. Gard. 37(1): 64. $1950 . \quad$ Fig. 2g-i

Arbustos de até $3 \mathrm{~m}$ de alt., ramos glabrescentes a hirtelos, glândulas ausentes. Folhas 16,5-24,5 × 6-10 cm, elípticas, base assimétrica, com 1-1,8 cm de diferença entre os lobos, arredondada ou raramente subcordada, ápice agudo ou aristulado, cartáceas quando secas, glândulas ausentes, glabras, esparsamente hirtas a hirtelas nas nervuras da face abaxial, margem eciliada, venação pinada, 7-11 nervuras de cada lado desenvolvidas ao longo da nervura principal; pecíolo 0,3-0,9 cm compr., glabrescente a hirtelo, alado em toda extensão. Espigas 3-8,5 cm compr., eretas; pedúnculo $0,5-1 \mathrm{~cm}$ compr., glabrescente a esparsamente hirtelo; raque glabra. Bráctea floral orbicular; estigma séssil. Drupas $1-1,5 \times 0,2-0,5$ $\mathrm{mm}$, oblongas, glabras.

Material examinado selecionado: Mata do Pezinho, 4.X.2007, fl., D. Araújo et al. 579 (UFP); 15.II.2008, fr., A. Alves-Araújo \& J.S. Marques 866 (UFP); Mata de Piedade, 10.I.2008, fr., A. Alves-Araújo et al. 794 (UFP).

Presente na América Central e América do Sul (Yuncker 1973; Steyermark \& Callejas-Posada 2003). No Brasil ocorre na Amazônia, Cerrado e Mata Atlântica, e no Nordeste está registrada para o Ceará e Bahia (Guimarães et al. 2012), sendo aqui registrada para Pernambuco. Comum na área, é diferenciada dos demais táxons por suas folhas elípticas de base fortemente assimétrica (com até $1,8 \mathrm{~cm}$ entre os lobos) e pecíolo alado.
6. Piper caldense C. DC, Linnaea 37: 343-344. 1872.

Fig. $2 \mathrm{j}-1$

Arbustos até $2,5 \mathrm{~m}$ de alt., glândulas negras presentes; ramos glabros. Folhas 14-19 × 4-7 $\mathrm{cm}$, elípticas, base assimétrica, com até $0,2 \mathrm{~cm}$ de diferença entre os lobos, cuneada, ápice agudo, cartáceas quando secas, glabras, margem eciliada, venação pinada, $6-8$ nervuras secundárias surgindo até à porção mediana da nervura principal; pecíolo $0,4-0,7 \mathrm{~cm}$, glabro, sulcado na base. Espigas $2,5-3$ $\mathrm{cm}$ compr., eretas; pedúnculo 0,7-1 cm compr., glabro; raque glabra. Bráctea floral orbicular a levemente triangular; estilete desenvolvido. Drupas 1-2 × 1-1,5 mm, globosas, glabras.

Material examinado: Mata dos Macacos, 8.II.2008, fr., J.S. Gomes et al. 312 (UFP); Mata de Piedade, 6.V.2009, fr., T.A. Pontes 189 (UFP).

Material examinado adicional: BRASIL. RIO GRANDE DO NORTE: Pipa, 26.IX.2011, fl. e fr., $A$. Melo et al. 973 (UFP).

Endêmica do Brasil, presente na Caatinga, Cerrado e Mata Atlântica. No Nordeste está registrada para os estados do Ceará, Paraíba, Pernambuco, Alagoas e Bahia (Guimarães et al. 2012), sendo nesse trabalho, sua distribuição ampliada para o estado do Rio Grande do Norte. $\mathrm{Na}$ USJ foi encontrada no interior dos fragmentos, próximo a áreas alagadas ou nas bordas florestais. Diferencia-se das demais pelas poucas nervuras secundárias (geralmente 6-8) ascendentes, inflorescências geralmente curtas, além de ser a única espécie da área com estilete desenvolvido.

7. Piper hispidum Sw., Prodr.: 15. 1788.

Fig. 3a-d

Arbustos de até $3 \mathrm{~m}$ de alt.; ramos pilosos. Folhas 11,5-17 × 4,5-7,7 cm, elípticas a ovadas, base assimétrica, com $0,4-0,6 \mathrm{~cm}$ de diferença entre os lobos da base, obtusa, ápice acuminado, cartáceas quando secas, glândulas marrons presentes em ambas as faces, face adaxial híspida, opaca quando seca, face abaxial hirtela, margem ciliada, venação pinada, 6-8 nervuras secundárias surgindo até próximo à porção mediana da nervura principal; pecíolo 0,4-0,7 cm compr., piloso, sulcado na base. Espigas 9-12 cm compr., eretas; pedúnculo 0,7-1 cm compr., híspido; raque híspida. Bráctea floral triangular a orbicular; estigma séssil, inserido em depressão apical no fruto. Drupas $0,5-1$ $\times 0,3-0,5 \mathrm{~mm}$, oblongas, puberulentas.

Material examinado: Engenho D'água, 24.V.2003, fr., A. Melquiades \& G.J. Bezerra 182 (UFP); Mata dos Macacos, 23.XII.2011, fl., A. Melo et al. 967 (UFP); 


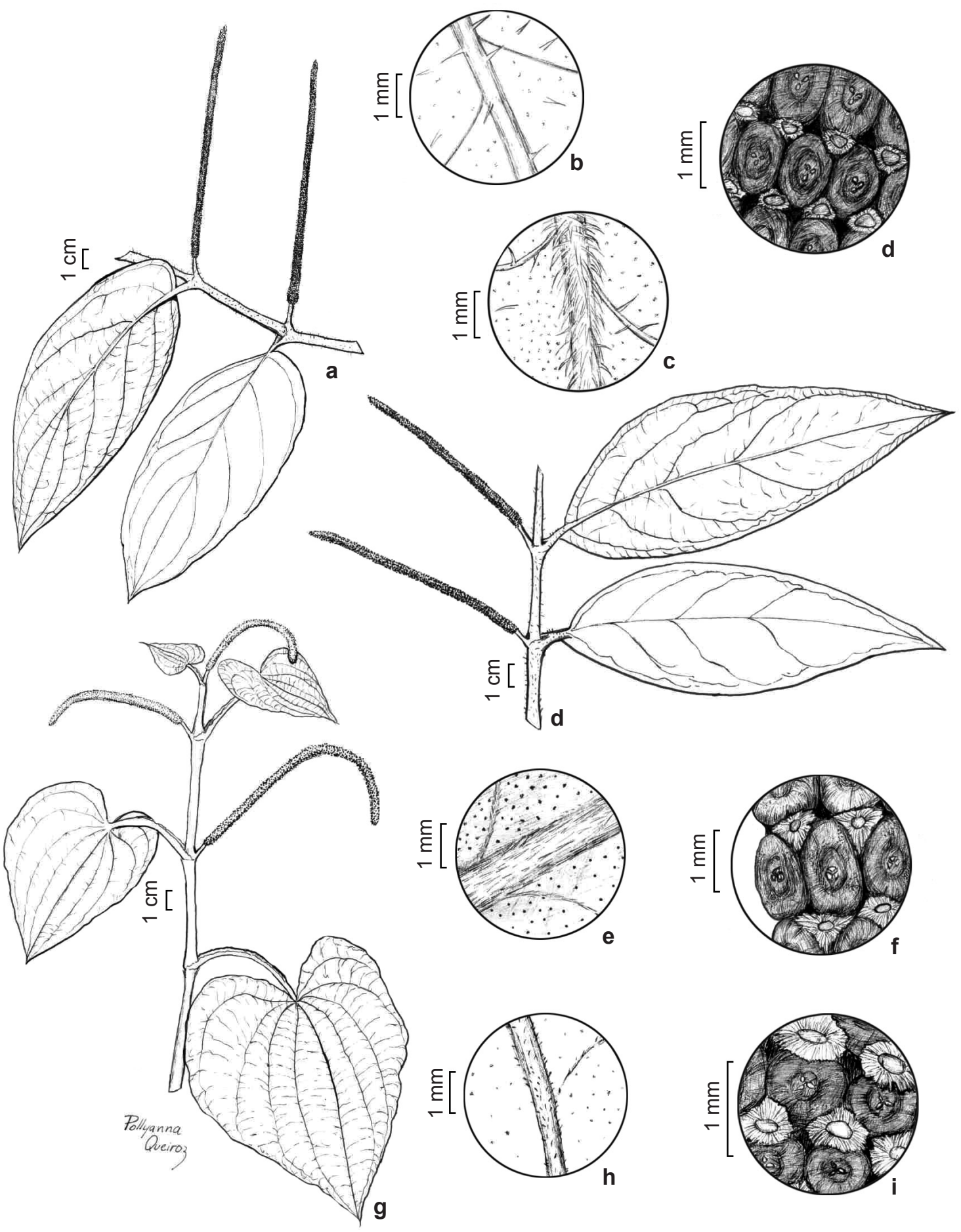

Figura 3 - a-d. Piper hispidum Sw. - a. ramo; b. face adaxial da folha; c. face abaxial da folha; d. frutos. e-g. Piper hostmannianum (Miq.) C.DC. - e. ramo; f. face abaxial da folha; g. fruto. h-j. Piper marginatum Jacq. - h. ramo; i. face abaxial da folha; j. fruto. (a-d. Melo 967; e-g. Alves-Araújo 648; h-j. Melo 966).

Figura 3 - a-d. Piper hispidum Sw. - a. shoot; b. above surface; c. beneath surface; d. fruits. e-g. Piper hostmannianum (Miq.) C.DC. e. Shoot - f. beneath surface; g. fruits. h-j. Piper marginatum Jacq. - h. shoot; i. beneath surface; j. fruits. (a-d. Melo 967; e-g. AlvesAraújo 648; h-j. Melo 966). 
Mata do Pezinho, 11.I.2008, fr., A. Alves-Araújo et al. 824 (UFP); Mata de Piedade, 20.XII.2007, fr., A. AlvesAraújo \& D. Araújo 740 (UFP).

Material adicional examinado: BRASIL. PARAÍBA: Sapé, RPPN Fazenda Pacatuba, 4.V.2001, fl., E. César 187 (JPB).

Ocorre nas Ilhas Caribenhas, América Central e América do Sul (Trelease \& Yuncker 1950). No Brasil está presente na Amazônia, Cerrado e Mata Atlântica, e no Nordeste pode ser encontrada no Ceará, Pernambuco, Alagoas e Bahia (Guimarães et al. 2012), sendo aqui sua distribuição ampliada para o estado da Paraíba. Na USJ é comum no interior dos fragmentos além de áreas de borda. Diferenciase por possuir folhas da face adaxial ásperas ao toque, e espigas eretas com 9-12cm compr.

\section{Piper hostmannianum (Miq.) C. DC. in DC.,} Prodr. 16(1): 287. 1869.

Fig. 3e-g

Arbustos escandentes até $1,5 \mathrm{~m}$ alt.; ramos pilosos. Folhas 14,5-19,7 × 6,5-8 cm, ovadas, base assimétrica, com até $0,3 \mathrm{~cm}$ de diferença entre os lobos, arredondados, ápice agudo, cartáceas quando secas, glândulas marrons presentes na face abaxial, face adaxial lustrosa quando seca, face abaxial pilosa, margem eciliada, venação pinada, 6-8 nervuras secundárias ascendentes e surgindo até próximo à porção mediana da nervura principal e outras nervuras de menor calibre seguindo em direção à margem; pecíolo 0,4-1,2 cm compr., piloso, sulcado na base. Espiga 5,3-11,8 cm compr., ereta; pedúnculo 0,4-0,6 cm compr., piloso; raque glabra à pilosa. Bráctea floral triangular a arredondada, subpeltada; estigma séssil, inserido numa depressão apical do fruto. Drupas de até 0,5-1 $\times 0,5-0,7 \mathrm{~mm}$, oblongas, puberulentas.

Material examinado: Mata de Zambana, 18.X.2007, fl. e fr., A. Alves-Araújo et al. 648 (UFP).

Material adicional: BRASIL. AMAPÁ: Campo Grande, 25.X.1979, fr., D.F. Austin et al. 7218 (INPA, RB). PARÁ: Oriximiná, Rio Cachorro, 20.VI.1980, fr., G. Martinelli et al. 7120 (INPA, RB).

Distribuída na Venezuela, Guiana, Suriname, Guiana Francesa e Amazônia Brasileira (Callejas-Posada et al. 2007; Guimarães et al. 2012), onde está presente nos estados do Amapá, Amazonas, Acre e Pará (Guimarães et al. 2012), sendo aqui registrada pela primeira vez para o Nordeste. Rara na USJ e coletada em área de borda próximo à local alagado. Distingue-se das demais pelo hábito escandente, as folhas lustrosas na face adaxial, face abaxial com pontuações, além do padrão de venação.
9. Piper marginatum Jacq., Icon. pl. rar. 2(11): t. 215. 1792.

Fig. $3 \mathrm{~h}-\mathrm{j}$

Arbustos de até $3 \mathrm{~m}$ de alt.; ramos glabros. Folhas 8,5-17 ×6-14 cm, cordadas, base simétrica, cordada, ápice acuminado, membranáceas quando secas, glândulas marrons por vezes presentes, glabrescentes a híspidas, margem ciliada, venação palmada, 9-11 nervuras; pecíolo 1,5-4 cm compr., glabro ou esparsamente piloso, alado em quase toda extensão. Espigas 10-16 cm compr., arqueadas; pedúnculo 0,6-1,5 cm compr., glabro; raque glabra. Bráctea floral orbicular ou levemente triangular; estigma séssil, inserido em depressão apical no fruto. Drupas até $1 \times 1 \mathrm{~mm}$, oblongas, glabras.

Material examinado selecionado: Córrego do Urubu, 17.IV.2008, fl., L.M. Nascimento \& G. Batista 682 (UFP); Mata Chave, 24.XI.2009, fr., E. Pessoa \& J.A.N. Souza 154 (UFP); Mata dos Macacos, 23.XII.2011, fl., $A$. Melo et al. 966 (UFP); Mata do Pezinho, 25.V.2005, fl., J.S. Gomes \& H.C.H. Silva 95 (PEUFR, UFP); Mata de Piedade, 16.X.2002, fl. e fr., G.J. Bezerra \& M.J. Silva 48 (PEUFR, UFP); Mata de Santa Helena, 8.X.2003, fl. e fr., I.M.M. Sá e Silva 186 (PEUFR, UFP).

Ocorre desde a América Central, Caribe até o Equador (Yuncker 1972). No Brasil está presente nos domínios da Amazônia, Caatinga, Cerrado e Mata Atlântica, e no Nordeste do Ceará à Bahia (Guimarães et al. 2012). Na USJ é uma espécie comum na borda dos fragmentos e em áreas de capoeira e diferencia-se das demais por folhas de base simétrica, cordiformes e venação palmada.

\section{Agradecimentos}

Aos financiadores da pesquisa: CNPq, U.S. National Science Foundation (DEB-0946618), Velux Stiftung, Beneficia Foundation e FACEPE; além do projeto "Sustentabilidade de remanescentes de Floresta Atlântica em Pernambuco e suas implicações para a conservação e desenvolvimento local", que contou com apoio do BMBF-Alemanha. Aos curadores dos herbários visitados e à Pollyanna Queiroz, as ilustrações de Piperaceae.

\section{Referências}

Alves-Araújo, A. \& Alves, M. 2010. Flora da Usina São José, Igarassu, Pernambuco: Sapotaceae. Rodriguésia 61: 303-318.

Alves-Araújo, A.; Araújo, D.; Marques, J.; Melo, A.; Maciel, J.; Irapuã, J.; Pontes, T.; Lucena, M.F.A.; Bocage, A.L. \& Alves, M. Diversity of angiosperms in fragments of Atlantic Forest in the State of Pernambuco, Northeastern Brazil. Bioremediation, Biodiversity \& Bioavailability 2: 14-26 
Amorim, B.S. \& Alves, M. 2011. Flora da Usina São José, Igarassu, Pernambuco: Myrtaceae. Rodriguésia 62: 499-514.

Angiosperm Phylogeny Group -APG III. 2009. An update of the Angiosperm phylogeny group classification for the orders and families of flowering plants: APG III. Botanical Journal of the Linnean Society 161: 105-121.

Araujo, D. \& Alves, M. 2010. Climbing plants of a fragmented area of lowland Atlantic Forest, Igarassu, Pernambuco (Northeastern Brazil). Phytotaxa 8: 1-24.

Araújo, D. \& Alves, M. 2013. Flora da Usina São José, Iagarassu, Pernambuco: Passifloraceae s.s. Rodriguésia 64 (in press).

Barros, F. \& Araújo, A.A.M. 2012. Aristolochiaceae. In: Forzza, R.C. et al. (eds.). Lista de espécies da flora do Brasil. Jardim Botânico do Rio de Janeiro. Disponível em <http://floradobrasil.jbrj.gov. br/2012/FB000054>. Acesso em 30 Abr 2012.

Barroso, G.M.; Guimarães, E.F.; Ichaso, C.L.F.; Costa, C.G. \& Peixoto, A.L. 1978. Sistemática de angiospermas do Brasil. Vol. 1. Ed. Universidade de São Paulo, São Paulo. 255p.

Buril, M.T. \& Alves, M. 2011. Flora da Usina São José: Convolvulaceae. Rodriguésia 62: 93-105.

Callejas-Posada, R.; Görts-van Rijn, A.R.A. \& Steyermark, J.A. 2007. Piperaceae. In: Funk, V.; Hollowell, T.; Berry, P.; Kelloff, C. \& Alexander, S.N. (eds.). Checklist of the plants of the Guiana Shield (Venezuela: Amazonas, Bolivar, Delta Amacuro; Guyana, Surinam, French Guiana). National Museum of Natural History, Washington. Pp. 458-466.

Capellari Jr., L. 2002. Aristolochiaceae. In: Wanderley, M.G.L.; Shepherd, G.J. \& Giulietti, A.M. (eds.). Flora fanerogâmica do estado de São Paulo. Vol. 2. HUCITEC, São Paulo. Pp. 39-49.

Castro, M.J.P.; Silva, P.H.S. \& Pádua, L.E.M. 2010. Potencial de extratos de frutos frescos e desidratados de Piper tuberculatum Jacq. (Piperaceae) no desenvolvimento da largata-do-cartucho do milho. Magistra 22: 90-96.

Di Stasi, L.C.; Himura-Lima, C.A.; Mariot, A.; Portilho, W.C. \& Reis, M.S. 2002. Piperales medicinais. In: Di Stasi, L.C. \& Himura-Lima, C.A. (eds.). Plantas medicinais na Amazônia e na Mata Atlântica. $2^{\mathrm{a}}$ ed. Ed. UNESP, São Paulo. Pp. 120-138.

Gomes-Costa, G.A. \& Alves, M. 2012. Flora da Usina São José, Igarassu, Pernambuco: Cucurbitaceae. Rodriguésia, 63:817-829

González, F. 1999. Inflorescence morphology and the systematics of Aristolochiaceae. Systematics and Geography of Plants 68: 159-172.

González, F. 2004. Aristolochiaceae. In: Smith, N.; Mori, S.A.; Henderson, A.; Stevenson, D.W. \& Heald, S.V. (eds.). Flowering plants of the Neotropics. Princeton University Press, New Jersey. Pp. 31-33.
Guimarães, E.F.; Carvalho-Silva, M.; Monteiro, D.\& Medeiros, E. 2012. Piperaceae. In: Forzza, R.C. et al. (eds.). Lista de espécies da flora do Brasil. Jardim Botânico do Rio de Janeiro. Disponível em <http:// floradobrasil.jbrj.gov.br/2012/FB000190>. Acesso em 30 Abr 2012.

Hoehne, F.C. 1942. Aristolochiaceas. In: Hoehne, F.C. (ed.). Flora Brasilica 15: 1-141.

Huber, H. 1993. Aristolochiaceae. In: Kubitzi, K.; Rohwer, J.G.; Bittrich, V. (eds.). Flowering plants: Dicotyledons. Magnoliid, Hamamelid and Caryophyllid families. Vol. 2. Springer-Verlag Berlin, Heidelberg. Pp. 129-137.

Lemos, R.P.L.; Mota, M.C.S.; Chagas, E.C.O. \& Silva, F.C. 2010. Checklist. Flora de Alagoas: Angiospermas. Instituto de Meio Ambiente de Alagoas, Herbário MAC, Maceió. 141p.

Lima, R.K.; Cardoso, M.G.; Moraes, J.C.; Melo, B.A.; Rodrigues, V.G. \& Guimarães, P.L. 2009. Atividade inseticida do óleo essencial de pimenta longa (Piper hispidinervum C. DC.) sobre lagarta-do-cartucho do milho Spodoptera frugiperda (J. E. Smith, 1797) (Lepidoptera: Noctuidae). Acta Amazônica 39: 377-382.

Melo, A.; Alves-Araújo, A. \& Alves, M. 2010. Burmanniaceae e Gentianaceae da Usina São José, Igarassu, Pernambuco. Rodriguésia 61: 431-440.

Melo, A.; Amorim, B.S.; García-González, J.D.; Souza, J.A.N.; Pessoa, E.; Mendonça, E.; Chagas, M.; Alves-Araujo, A. \& Alves, M. 2011. Updated floristic inventory of the angiosperms of the Usina São José, Igarassu, Pernambuco, Brazil. Revista Nordestina de Biologia 20: 3-26.

Mendes, M.; Gomes, P. \& Alves, M. 2010. Floristic inventory of a zone of ecological tension in the Atlantic Forest of Northeastern Brazil. Rodriguésia 61: 669-676.

Mori, S.A.; Mattos-Silva, L.A.; Lisboa, G. \& Coradin, L. 1989. Manual de manejo do herbário fanerogâmico. $2^{\mathrm{a}}$ ed. CEPLAC/CEPEC, Ilhéus. 104p.

Nee, M. 2004. Piperaceae. In: Smith, N.; Mori, S.A.; Henderson, A.; Stevenson, D.Wm.; Heald, S. (eds.). Flowering plants of the neotropics. Princenton University Press, New Jersey. Pp. 296-297.

Oliveira, S.O. \& Alves, M. 2012. Flora da Usina São José, Igarassu, Pernambuco: Lauraceae. Rodriguésia 63: 689-703.

Pessoa, E. \& Alves, M. 2012. Flora da Usina São José, Igarassu, Pernambuco: Orchidaceae. Rodriguésia 63: 341-356.

Pfeifer, H.W. 1966. Revision of the North and Central American hexandrous species of Aristolochia (Aristolochiaceae). Annals of The Missouri Botanical Garden 53: 115-196.

Pontes, T. \& Alves, M. 2010. Flora da Usina São José, Igarassu, Pernambuco: Araceae. Rodriguésia 61: 689-704. 
Quijano-Abril, M.A.; Callejas-Posada, R. \& MirandaEsquivel, D.R. 2006. Areas of endemism and distribution patterns for Neotropical Piper species (Piperaceae). Journal of Biogeography 33: 12661278.

Reveal, J.L. 2012. An outline of a classification scheme for extant flowering plants. Phytoneuron 2012: 1-221.

Samain, M.S.; Mathieu, G.; Wanke, S.; Neinhuis, C. \& Goetghebeur, P. 2008. Verhuellia revisitedunravelling its intricate taxonomic history and a new subfamilial classification of Piperaceae. Taxon 57: 583-587.

Silva, F.O.; Lourenço, A.R.L.; Pessoa, M.C.R. \& Alves, M.V. 2012. Flora da Usina São José, Igarassu, Pernambuco: Ochnaceae e Quiinaceae. Rodriguésia 63: 1133-1138.

Silva, W.C.; Ribeiro, J.A.; Souza, H.E.M. \& Corrêa, R.S. 2007. Atividade inseticida de Piper aduncum L. (Piperaceae) sobre Aetalion sp. (Hemiptera: Aetalionidae), praga de importância econômica no Amazonas. Acta Amazonica 37: 293-298.

Steyermark, J.A. \& Callejas-Posada, R. 2003. Piperaceae. In: Steyermark, J.A.; Berry, P.E.; Yatskievych, K.; Holst, B. (eds.). Flora of the Venezuela Guayana. Vol. 7. Missouri Botanical Garden Press, St. Louis. Pp. 681-738.

Tebbs, M.C. 1993. Piperaceae. In: Kubitzi, K.; Rohwer, J.G.; Bittrich, V. (eds.). Flowering plants: Dicotyledons. Magnoliid, Hamamelid and
Caryophyllid families. Vol. 2. Springer-Verlag, Berlin Heidelberg. Pp. 516-520

Thiers, B. 2012. Index Herbariorum: A global directory of public herbaria and associated staff. New York Botanical Garden's Virtual Herbarium. Disponível em <http://sweetgum.nybg.org/ih/>. Acesso em 30 Abr 2012.

Trelease, W. \& Yuncker, T.G. 1950. The Piperaceae of Northern South America. 2 vols. University of Illinois Press, Urbana. 838p.

Trindade, M.B.; Lins-e-Silva, A.C.B.; Silva, H.P.; Figueira, S.B. \& Schessl, M. 2008. Fragmentation of the Atlantic rainforest in the northern coastal region of Pernambuco, Brazil: Recent changes and implications for conservation. Bioremediation, Biodiversity and Bioavailability 2: 5-13.

Wanke, S.; Samain, M.S.; Vanderschaeve, L.; Mathieu, G.; Goetghebeur, P. \& Neinhuis, C. 2006. Phylogeny of the genus Peperomia (Piperaceae) inferred from the trnK/matK region (cpDNA). Plant Biology 8: 93-102

Yuncker, T.G. 1972. The Piperaceae of Brazil. Piper, Group I, II, III, IV. Hoehnea 2: 19-366.

Yuncker, T.G. 1973. The Piperaceae of Brazil II. Piper, Group V: Ottonia, Pothomorphe, Sarcorhachis. Hoehnea 3: 29-284.

Yuncker, T.G. 1974. The Piperaceae of Brazil III. Peperomia: Taxa of uncertain status. Hoehnea 4: 71-413.

\section{Lista de Exsicatas}

Agra, M.F. 503 (6). Albuquerque, N.A. 639 (4). Albuquerque, U.P. 27 (4). Alves-Araújo, A. 484,784, 866 (3), 485, 721 (4), 572, 794, 866 (5), 740, 793,824 (7), 648 (8). Alves, M. s.n. UFP 11572 (1). Alves, S. s.n. UFP 18588 (9). Amazonas, J.P. s.n. UFP 4574, s.n. UFP 8896 (9). Andrade, I.M. s.n. UFP 1188, 25 (4), 15 (9). Andrade-Lima, D. 57-2780 (3). Araújo, A.A.M. 315 (1), 354 (2). Araújo, D. 579 (5), 602 (2). Araújo, F. 11 (5). Arbo, M.M. 7856 (5). Ataide, M. 341, 342 (9). Austin, D.F. 7218 (8). Baracho, G.S. 535 (9). Baracho, G.S. \& Siqueira-Filho, J.A. 206, 520 (1). Barbosa, D. s.n. UFP 43489. Barbosa, T.C.L. 3 (4). Barreto, I.S.S. s.n. UFP 32488 (4). Barreto, R. 83-081, 381 (5). Bedi, R. 95-1986 (4), 262 (7), 133 (9). Bezerra, G.J. 48 (9), 108 (2). Borges, M. s.n. IPA 52845 (5). Cano, O. s.n. IPA 74046 (4), 73 (9). César, E. 187 (7). Conceição, A.S. 8 (9). Cordeiro, A.P.R. 7 (4), s.n. IPA 81333 (9). Corrêa, M.A.C. s.n. UFP 3035 (1). Correia, I. 32 (4). Costa, J.T. 113-67 (2). Costa e Silva, M.B. 190, 1411 (9). Ehrendorrfer, F. s.n. IPA 44638 (5), s.n. IPA 44637 (9). Elesbão, A. 8 (5), s.n. UFP 6709, 1 (9). Eugenio, C. s.n. IPA 65092, 479 (9). Falcão, B.P.S. 91 (4). Falcão, J.I.A. 951 (3). Falcão, M. 19 (9). Félix, L.P. s.n. UFP 40632, 9822 (6). Ferreira, C. 67 (4). Gomes, J. 312 (6), 95, 211 (9). Gomes, P. 650 (4). Griz, L. \& Lopes, A. s.n. UFP 10945 (1). Guedes, M.L. 2217 (9). Harley, R.M. 15335, 17157 (5), 16576, 17501 (7). Lemos, J.M. s.n. UFP 7741 (4). Lima, A. 37-68 (3), 392-68 (5), 57-2770 (6). Lima, A.K. s.n. IPA 70289 (4). Lima, C.L. s.n. UFP 31052 (9). Lima, R.S. 9 (6). Lima, V.C. 537 (9). Lira, O.C. $42-67$ (4). Locatelli, E.S. s.n. UFP 39456 (4). Locatelli, E.S. \& Medeiros, P. s.n. UFP 39140, 39141 (1). Lucena, M.F.A. 1439 (4). Lyra-Lemos, R.P. 5881, 7848 (9). Machado, I.C.S. s.n. UFP 4341 (1). Maia, D.F. s.n. UFP 7.440 (9). Mariz, G. 183, s.n. UFP 5391 (1). Mariz, G. 183 (4), 2056 (6). Marques, J.S. 157 (9). Martinelli, G. 7120 (8). Mayo, S.J. 1083 (5), 405 (6), 1085 (7). Mc Dowell, T. 3323 (8). Melo, A. 104 (3), 133 (4), 378 (6), 967 (7), 966 (9). Melquíades, A. 182 (7), 178 (9), 199 (1). Mendes, K. 315,341 (3), 338 (5). Miranda, A.M. s.n. HST 16863 (1). Monte, N. 2 (9). Montenegro, P. 17 (9). Moraes, M. 11 (9). Moura, E. 70 (9). Nascimento, L.M. 442, 698 (5), 682 (9). Ojima, P.Y. 34, 41 (9). Oliveira, A. s.n. IPA 51372 (5). Oliveira, J.B.S. 123 (4), 98, 121, 164 (9). Oliveira, M. 626, 1265 (5), 673 (7), 1192, 1264 (9). Pereira, R. s.n. IPA 66150 (4), s.n. IPA 56701 (5), 1 (9). Pessoa, E. 154 (9), 227 (2). Pickel, D. B. 2085 (3), 405, 2444 (4), 4088, 4326 (5), 1873 (7), 166 (9). Pietrobom-Silva, M.R. 3431 (5). Pinheiro, R.S. 2203 (3), s.n. IPA 26397 (6). Pontes, T.A. 189 (6). Pontual, I 66-281 (3). Porto, K.C. s.n. UFP 4373 (3), s.n. UFP 4261 (6). Ribeiro, M. 11 (4). Rocha, E.A. 205 (2). Sá e Silva, I.M.M. 186 (9). Santana, C.A. s.n. IPA 57062 (4). Santos, A.C.M. s.n. UFP 15987 (9). Santos, P.A. 34 (9). Santos, T.S. 2696,3767 (5). Schmidt, S. 89 (9). Silva, A.G. 58, 335 (5). Silva, A. 25 (4), s.n. IPA 58931, s.n. IPA 61611 (9). Silva, E.S. 16 (9). Silva, H.C.H. 99 (9). Silva, L.M.B. s.n. UFP 11440 (4). Silva, S.I. s.n. UFP 4405 (4), s.n. UFP 4216 (9). Silva, V.A. s.n. UFP 9565 (4). Siqueira-Filho, J.A. 1197 (3), 449, 1079, 1372 (6). Sobral-Leite, M. 533, 811, 1246 (4), 398, 725 (9). Staviski, M.N.R. 395 (9). Tschá, M. 5 (6). Tenório, E. 66-204 (5). Vasconcelos-Sobrinho, J. s.n. IPA 76 (4), s.n. IPA 573 (5). Viana, A. 231 (6). Viana, A.M.B. s.n. UFP 40633 (6). Vicente, M.M. 11 (9). 